Case Report

\title{
Unusual Presentation of Cutaneous Leishmaniasis: Ocular Leishmaniasis
}

\author{
Masoud Doroodgar, ${ }^{1}$ Moein Doroodgar, ${ }^{1}$ and Abbas Doroodgar ${ }^{2}$ \\ ${ }^{1}$ School of Medicine, Shahid Beheshti University of Medical Sciences, Tehran, Iran \\ ${ }^{2}$ Department of Medical Parasitology, Kashan University of Medical Sciences, Kashan, Iran \\ Correspondence should be addressed to Abbas Doroodgar; adoroudgar@gmail.com
}

Received 2 October 2016; Accepted 25 December 2016; Published 22 January 2017

Academic Editor: Paola Di Carlo

Copyright (C) 2017 Masoud Doroodgar et al. This is an open access article distributed under the Creative Commons Attribution License, which permits unrestricted use, distribution, and reproduction in any medium, provided the original work is properly cited.

\begin{abstract}
The leishmaniases are parasitic diseases that are transmitted to humans by infected female sandflies. Cutaneous leishmaniasis (CL) is one of 3 main forms of the disease. CL is the most common form of the disease and is endemic in many urban and rural parts of Iran and usually caused by two species of Leishmania: L. major and L. tropica. We report a case of unusual leishmaniasis with 25 lesions on exposed parts of the body and right eyelid involvement (ocular leishmaniasis). The patient was a 75-year-old male farmer referred to health care center in Aran va Bidgol city. The disease was diagnosed by direct smear, culture, and PCR from the lesions. PCR was positive for Leishmania major.
\end{abstract}

\section{Introduction}

Leishmaniasis is a disease caused by protozoan parasites of the genus Leishmania and transmitted by the bite of infected female phlebotomine sandflies. The disease can be seen in three forms: cutaneous, mucocutaneous, and visceral leishmaniasis [1-3]. Leishmaniasis is endemic in 98 countries with more than 350 million people at risk. Leishmaniasis is associated with environmental changes such as deforestation, building of dams, irrigation schemes, and urbanization [4]. The cutaneous leishmaniasis (CL) with 1.5 million new cases per year is the most common form of the disease and causes skin lesions, mainly ulcers, on exposed parts of the body $[3,4]$.

In the old and new world, up to $90 \%$ of cases of CL occur in Afghanistan, Algeria, Iran, Saudi Arabia, and Syrian Arab Republic and in Bolivia, Brazil, Colombia, Nicaragua, and Peru [3]. Both form of rural and urban CL; zoonotic CL (ZCL) and anthroponotic CL (ACL) were seen in Iran. CL with about annually 20,000 new cases is considered one of the most important parasitic diseases in Iran. In addition, sometimes there are a few unusual and atypical clinical forms [5-10].

Here a case of unusual leishmaniasis (ocular leishmaniasis) from endemic area of Aran va Bidgol (Isfahan province, central Iran) with 25 lesions on exposed parts of the body and eight lesions on the face and right eyelid involvement $(\mathrm{OL})$ is reported. The disease was diagnosed by direct smear, culture of lesion, and RAPD-PCR technique for identifying the leishman agent causative. The PCR product was compared to reference stocks: L. major (MHOM/IR/75/ER) and $L$. tropica (MHOM/IR/IR/99) and the results were obtained. The data related to the patient was analyzed by descriptive statistics and bands of PCR product were compared to the standard marker (XIV) strains.

\section{Case Report}

A 75-year-old man with complaint of lesions extended on the hands, legs, face, and upper lid of right eye was referred to a health center of Kashan.

He was a farmer in Aran va Bidgol city from Isfahan province in the center of Iran. The patient about his infection said, sandflies have bitten him when referring to the farm for irrigation on the nights in the season of sandflies activity.

Based on questioning, the patient described the appearance of 25 multiple nodular, ulcerative, and crusted lesions on his body. In physical examination the size of lesions was varied from a few millimeters to several centimeters. He had 

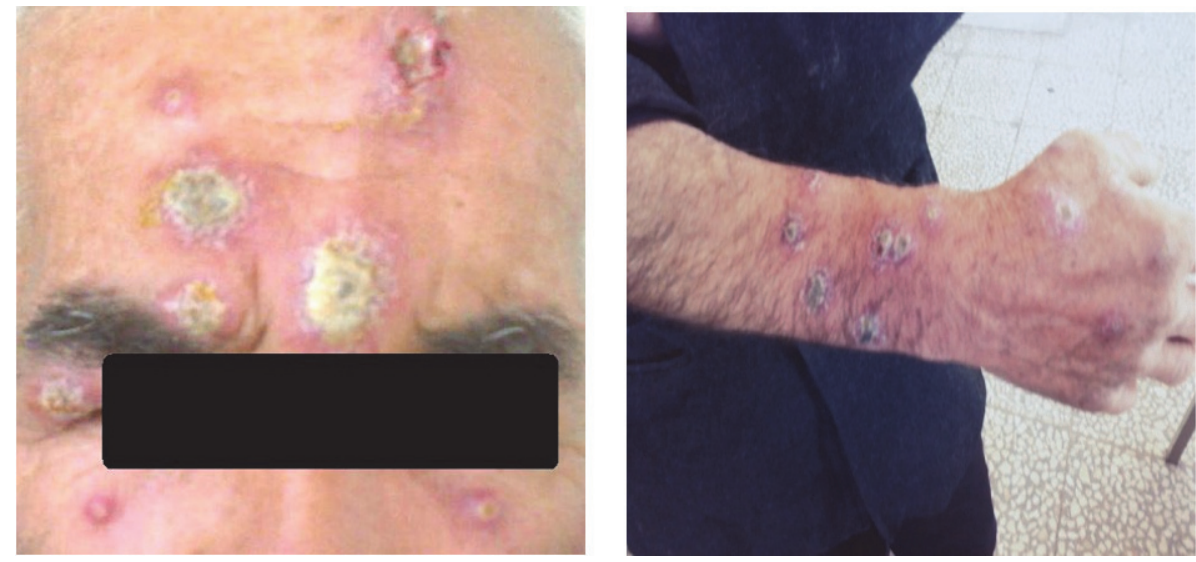

FIGURE 1: Patient with eight lesions on the face, right eyelid, and some lesions on the hands.

no particular medical history and systemic symptoms. There were eight lesions on the face that one of them was on the upper eyelid right eye (Figure 1).

In fact, the patient suffered from ocular leishmaniasis in addition to having multiple CL lesions. He had no fever and lesions were painless and his general condition was good.

Direct smears were prepared from the edge of skin lesions on different parts of body by using vaccinostyle and were fixed with pure methanol. Samples were stained by Giemsa $10 \%$ and examined under a light microscope and amastigote forms of Leishmania species were observed and CL was confirmed (Figure 2).

To identify the leishman agent causative of the disease materials of skin lesions cultured on RPMI-1640 medium plus 10\% FBS, DNA was extracted and PCR was performed. PCR was positive for Leishmania major (Figure 3).

The patient was treated intramuscularly by meglumine antimoniate (Glucantime).

\section{Discussion}

This report describes an unusual case of cutaneous leishmaniasis. The patient is residing and working in a rural area in the city of Aran va Bidgol (Isfahan province, located in the center of Iran). Many farms are located near or on the wild burrows in this county. According to the development of farms and human residency in wild rodents living area in the desert region of Aran va Bidgol, CL is the most common and the disease has been endemic. In recent years Aran va Bidgol county has been reported as an important zoonotic CL focus in Isfahan province [11]. Natural leishmanial infection caused by L. major in great gerbil (Rhombomys opimus) as reservoir and Phlebotomus papatasi sandfly as vector was confirmed in Aran va Bidgol [11].

In the case reported in the present study, the patient had no positive history of traveling to leishmaniasis endemic areas and there were 25 lesions on his body. Of these, 8 lesions were on the face that one of them was on the upper eyelid right

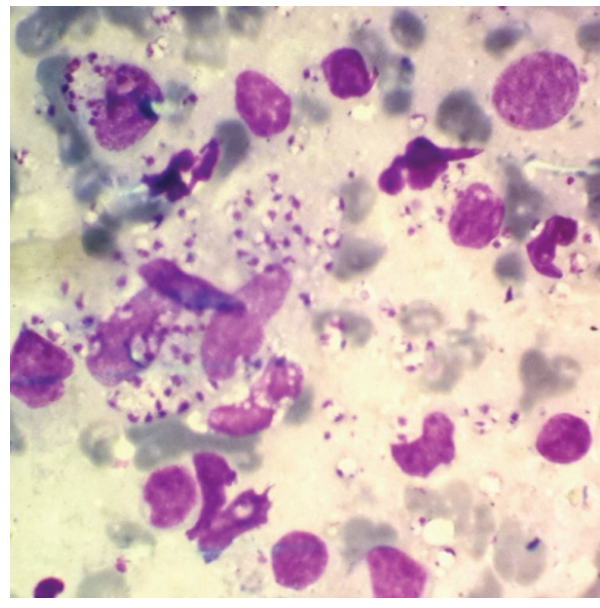

FIGURE 2: Direct smear was positive for leishman bodies (Giemsa stain).

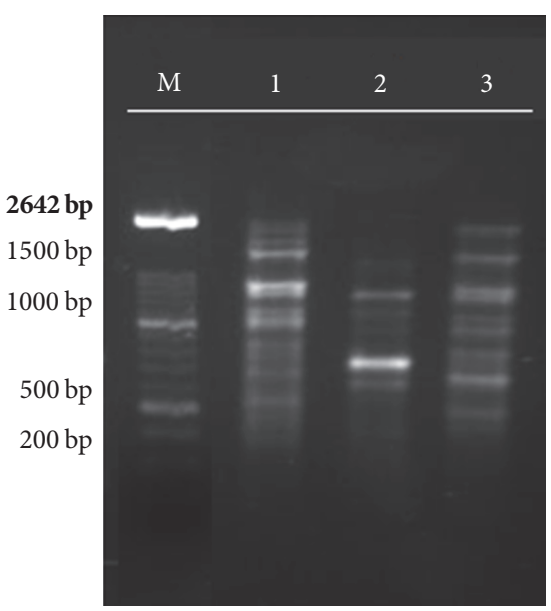

FIGURE 3: RAPD-PCR technique results by OPA4 primer in isolated strain and standards: M- marker (XIV $100 \mathrm{bp}$ ), (1) unknown strain, (2) L. tropica (MHOM/IR/IR/99), and (3) L. major (MHOM/IR/75/ER). 
eye and he was suffering from ocular leishmaniasis. Untreated ocular leishmaniasis may cause ophthalmologic side effects and can cause irreparable damage. In Iran, Modarres Zadeh et al. reported four cases of ocular leishmaniasis from 1950 to 2005. In Iran two of them ended in blindness [12]. Leishmania major with about $75 \%$ frequency is causative agent of zoonotic CL and is endemic in many rural areas of the Iran. CL caused by L. major is reported in 17 out of 31 provinces in Iran. Isfahan is one of the involved provinces $[13,14]$. In the present study, RAPD-PCR technique showed that causative agent was L. major. Ocular leishmaniasis is caused by different Leishmania species [12]. At the present time, most cases of atypical and OL lesions of cutaneous leishmaniasis which are reported from Iran are caused by L. major. Hajjaran et al. reported four unusual CL cases in which lesions are more located on hands, feet, and backs [8]. Moravvej et al. described four cases of unusual cutaneous leishmaniasis in which ulcers were located all over body, face, around eyes, chin, around upper lip, chest, and back [10]. Nasiri et al. described a case of CL on lower lip [15]. Ayatollahi et al. described a case of unusual cutaneous leishmaniasis involving a solitary lesion on the right eyelid (ocular leishmaniasis) [7]. Sadeghian et al. described a case of ocular leishmaniasis on lower and upper right eyelid margins [16]. Abrishami et al. reported a case of ocular leishmaniasis on right lower lid [17]. Jafari et al. described a case of OL on upper eyelid [18]. Nikandish et al. reported OL on the right eye [19]. In other parts of the world, several cases of unusual OL cases have been reported [20-26]. OL is a rare disease and involved $2.5 \%$ of cases with CL $[6,12]$. There is still no vaccine to prevent the disease and the drugs of treatment for all forms of leishmaniasis are pentavalent antimony compounds $[27,28]$.

Although cutaneous leishmaniasis is a self-limited disease [29], untreated OL can potentially be very serious for eyes. Since the early diagnosis and treatment of diseases can prevent the ophthalmologic side effects, ocular leishmaniasis should be considered and studied further in endemic areas of CL.

\section{Competing Interests}

The authors have no conflict of interests related to this work.

\section{Acknowledgments}

The authors thank and appreciate the patient for his cooperation and all who have collaborated with this case report.

\section{References}

[1] World Health Organization, Control of the Leishmaniases, World Health Organization, Geneva, Switzerland, 2010, http:// whqlibdoc.who.int/trs/WHO_TRS_949_eng.pdf.

[2] M. Service, Medical Entomology for Students, Cambridge University Press, Cambridge, UK, 4th edition, 2009.

[3] M. P. Barrett and S. L. Croft, "Management of trypanosomiasis and leishmaniasis," British Medical Bulletin, vol. 104, no. 1, pp. 175-196, 2012.
[4] World Health Organization, "Leishmaniasis Fact sheet," 2016, http://www.who.int/mediacentre/factsheets/fs375/en/.

[5] Ministry of Health and Medical Education, Care Guide Cutaneous Leishmaniasis in Iran, Center for Communicable Diseases Control, Tehran, Iran, 2012 (Persian), http://health .behdasht.gov.ir $/$ fkeyid $=\&$ siteid $=435 \&$ pageid $=53871 \&$ catid $=$ 289\&dview $=1189$.

[6] S. Veraldi, S. Bottini, N. Currò, and R. Gianotti, "Leishmaniasis of the eyelid mimicking an infundibular cyst and review of the literature on ocular leishmaniasis," International Journal of Infectious Diseases, vol. 14, supplement 3, pp. e230-e232, 2010.

[7] J. Ayatollahi, A. Ayatollahi, and S. H. Shahcheraghi, "Cutaneous leishmaniasis of the eyelid: a case report," Case Reports in Infectious Diseases, vol. 2013, Article ID 214297, 2 pages, 2013.

[8] H. Hajjaran, M. Mohebali, A. A. Akhavan, A. Taheri, B. Barikbin, and N. S. Soheila, "Unusual presentation of disseminated cutaneous leishmaniasis due to Leishmania major: case reports of four Iranian patients," Asian Pacific Journal of Tropical Medicine, vol. 6, no. 4, pp. 333-336, 2013.

[9] H. Mortazavi, M. Mohebali, Y. Taslimi et al., "Hoarseness as the presenting symptom of visceral leishmaniasis with mucocutaneous lesions: a case report," Iranian Journal of Parasitology, vol. 10, no. 2, pp. 296-300, 2015.

[10] H. Moravvej, M. Barzegar, S. Nasiri, E. Abolhasani, and M. Mohebali, "Cutaneous leishmaniasis with unusual clinical and histological presentation: report of four cases," Acta Medica Iranica, vol. 51, no. 4, pp. 274-278, 2013.

[11] A. Doroodgar, F. Sadr, M. R. Razavi, M. Doroodgar, M. Asmar, and M. Doroodgar, "A new focus of zoonotic cutaneous leishmaniasis in Isfahan Province, Central Iran," Asian Pacific Journal of Tropical Disease, vol. 5, supplement 1, pp. S54-S58, 2015.

[12] M. Modarres Zadeh, K. Manshai, M. Shaddel, and H. Oormazdi, "Ocular leishmaniasis review article," Iranian Journal of Ophthalmology, vol. 19, no. 3, pp. 1-5, 2006.

[13] M. R. Shirzadi, S. B. Esfahania, M. Mohebalia et al., "Epidemiological status of leishmaniasis in the Islamic Republic of Iran, 1983-2012," Eastern Mediterranean Health Journal, vol. 21, no. 10, pp. 736-742, 2015.

[14] M. R. Shirzadi, "Neglected tropical diseases innovative and intensified disease management leishmaniasis control," in Proceedings of the WHO Headquarters Report of the Consultative Meeting on Cutaneous Leishmaniasis, 2007.

[15] S. Nasiri, N. Mozafari, and F. Abdollahimajd, "Unusual presentation of cutaneous leishmaniasis: lower lip ulcer," Archives of Clinical Infectious Diseases, vol. 7, no. 2, pp. 66-68, 2012.

[16] G. Sadeghian, M. A. Nilfroushzadeh, S. H. Moradi, and S. H. Hanjani, "Ocular leishmaniasis: a case report," Dermatology Online Journal, vol. 11, no. 2, article 19, 2005.

[17] M. Abrishami, M. Soheilian, A. Farahi, and Y. Dowlati, "Successful treatment of ocular leishmaniasis," European Journal of Dermatology, vol. 12, no. 1, pp. 88-89, 2002.

[18] A. K. Jafari, M. Akhyani, M. Valikhani, Z. S. Ghodsi, B. Barikbin, and S. Toosi, "Bilateral cutaneous leishmaniasis of upper eyelids: a case report," Dermatology Online Journal, vol. 12, article no. 20, 2006.

[19] M. Nikandish, V. Mashayekhi Goyonlo, A. R. Taheri, and B. Kiafar, "Ocular leishmaniasis treated by intralesional amphotericin B," Middle East African Journal of Ophthalmology, vol. 23, no. 1, pp. 153-155, 2016. 
[20] M. P. Oliveira-Neto, V. J. Martins, M. S. Mattos, C. Pirmez, L. R. Brahin, and E. Benchimol, "South american cutaneous leishmaniasis of the eyelids: report of five cases in Rio de Janeiro State, Brazil," Ophthalmology, vol. 107, no. 1, pp. 169-172, 2000.

[21] M. S. Gurel, M. Ulukanligil, and H. Ozbilge, "Cutaneous leishmaniasis in Sanliurfa: epidemiologic and clinical features of the last four years (1997-2000)," International Journal of Dermatology, vol. 41, no. 1, pp. 32-37, 2002.

[22] M. Charif Chefchaouni, R. Lamrani, A. Benjelloune, M. El Lyacoubi, and A. Berraho, "Cutaneous leishmaniasis of the lid," Journal Francais d'Ophtalmologie, vol. 25, no. 5, pp. 522-526, 2002.

[23] E. Mencía-Gutiérrez, E. Gutiérrez-Díaz, J. L. Rodríguez-Peralto, and J. Monsalve-Córdova, "Old World eyelid cutaneous leishmaniasis: a case report," Dermatology Online Journal, vol. 11, no. 3, p. 29, 2005.

[24] A. Ul Bari and S. B. Rahman, "Many faces of cutaneous leishmaniasis," Indian Journal of Dermatology, Venereology and Leprology, vol. 74, no. 1, pp. 23-27, 2008.

[25] F. A. Ayele, Y. A. Wolde, T. Hagos, and E. Diro, "Ocular leishmaniasis presenting as chronic ulcerative blepharoconjunctivitis: a case report," Journal of Clinical \& Experimental Ophthalmology, vol. 6, article 395, 2015.

[26] A. Satici, B. Gurler, G. Aslan, and I. Ozturk, "Ocular involvement in cutaneous leishmaniasis four cases with blepharoconjunctivitis," European Journal of Epidemiology, vol. 19, no. 3, pp. 263-266, 2004.

[27] R. Kumar and C. Engwerda, "Vaccines to prevent leishmaniasis," Clinical \& Translational Immunology, vol. 3, no. 3, article no. e13, 2014.

[28] A. K. Haldar, P. Sen, and S. Roy, "Use of antimony in the treatment of leishmaniasis: current status and future directions," Molecular Biology International, vol. 2011, Article ID 571242, 23 pages, 2011.

[29] P. R. Machado, J. Ampuero, L. H. Guimarães et al., "Miltefosine in the treatment of cutaneous leishmaniasis caused by Leishmania braziliensis in Brazil: a randomized and controlled trial," PLOS Neglected Tropical Diseases, vol. 4, no. 12, p. e912, 2010. 


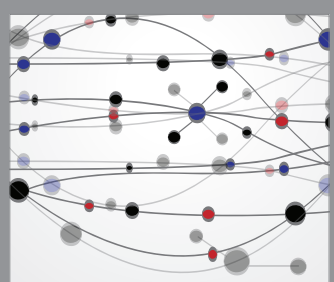

The Scientific World Journal
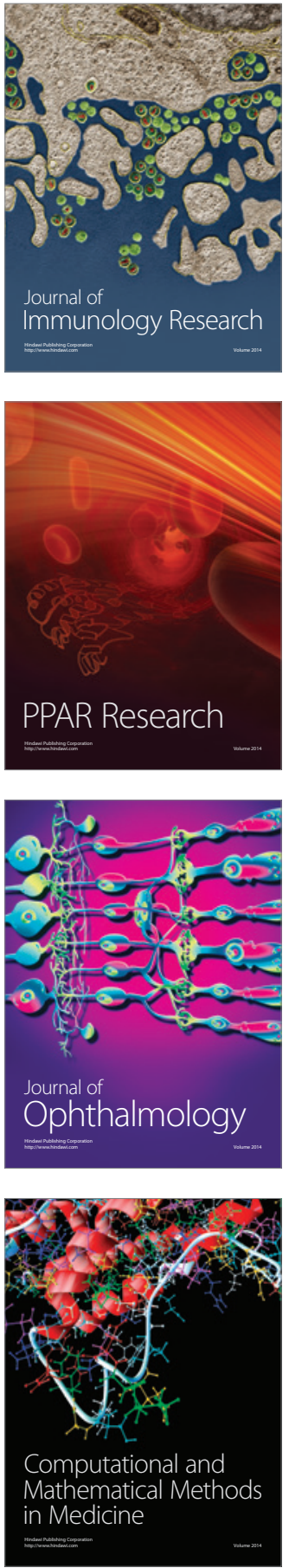

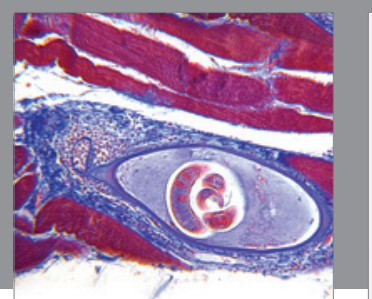

Gastroenterology Research and Practice
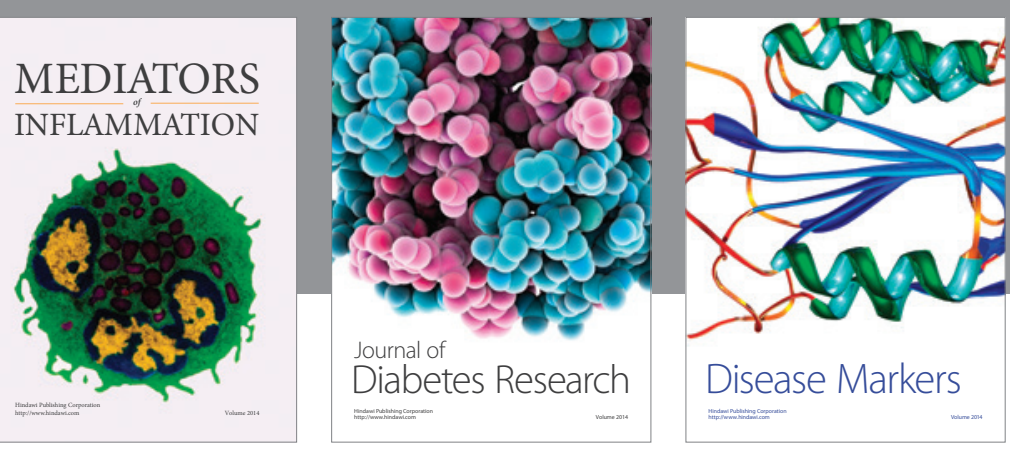

Disease Markers

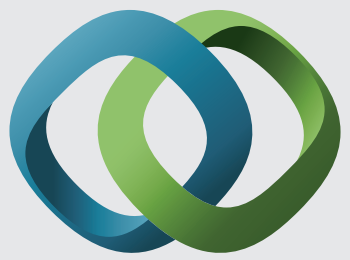

\section{Hindawi}

Submit your manuscripts at

https://www.hindawi.com
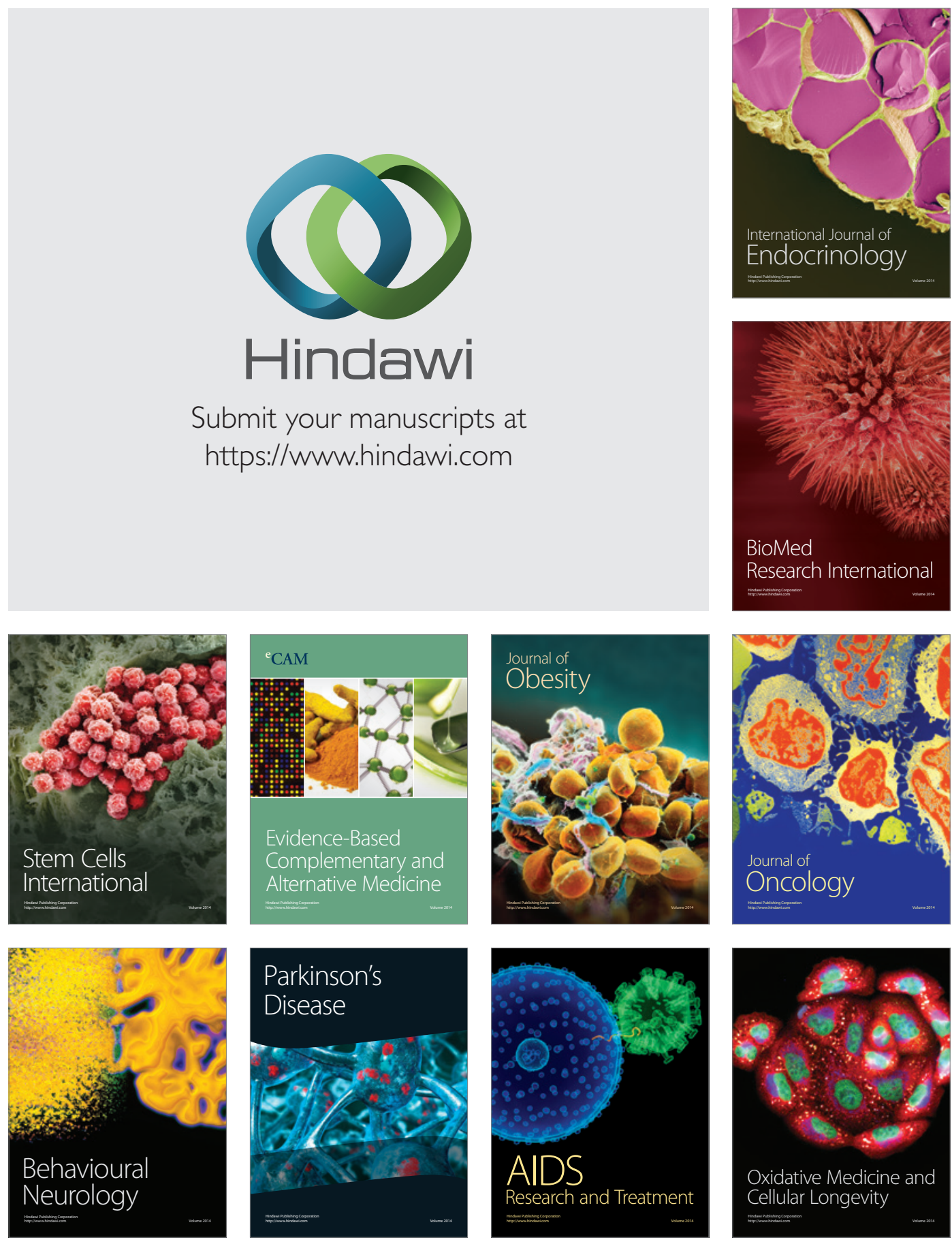